\title{
A POSIÇAO DO GEN Y3 NO CROMOSSOMO 2 DO MILHO *
}

\author{
E. A. GRANER \\ Escola Superior de Agricultura "Luiz de Queiroz" \\ Universidade de S. Paulo
}

INDICE

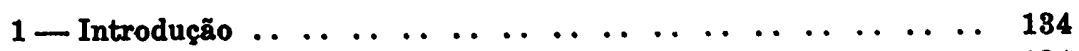

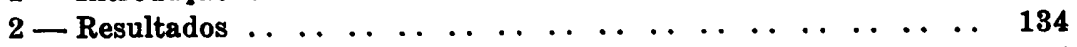

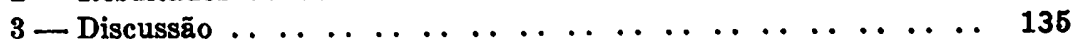

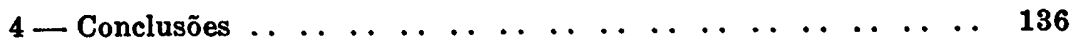

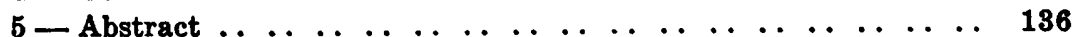

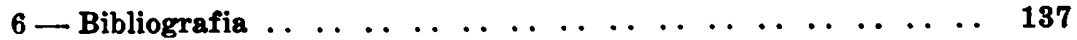

* Trabalho da Cadeira de Agricultura Especial e Genética Aplicada. Um resumo dêste trabalho foi apresentado à 2a. Reunião LatinoAmericana de Geneticistas e Fitoparasitologistas, reunida em Campinas e Piracicaba em 1952. 


\section{1 - INTRODUÇÃO}

A coloração amarela da semente de milho, comumente encontrada nas variedades cultivadas, depende da interação de 3 fatores genéticos: $Y 1, Y 3$ e $Y 7$ (6 e 9). Destes, o gen Y1 tem a sua posição bem definida no cromossomo 6 (2). A posição dos gens $Y 3$ e $Y 7$, respectivamente localizados nos grupos 2 e 7, está sendo estudada. Pretendemos, no presente trabalho, apresentar a posição do gen Y3 no cromossomo 2.

\section{2 - RESULTADOS}

Cruzamentos envolvendo os fatores $y 3, \lg 1$ e $g 12$, todos do grupo 2, foram planejados e executados, afim de se conhecer a posição do gen y3 no cromossomo 2 . Os resultados serão apresentados separadamente, para maior facilidade descritiva.

$$
2.1 \quad y 3-\lg 1
$$

Uma das linhagens contendo o gen $y 3$ foi cruzada com o teste $l g 1$, cromossomo 2. As plantas $\mathrm{F} 1$ foram autofecundadas e 7 espigas, segregando para F2, foram analisadas, estando os resultados contidos no quadro N. 1. A análise de ligação, tanto pela fórmula de soma, como pela fórmula de produto, foi feita para cada uma das espigas separadamente e também para o total delas.

Os resultados numéricos obtidos para o valor de ligação, pelas duas fórmulas, foram diferentes, razão pela qual procedemos a uma análise pelo teste $\chi 2$. Calculámos para cada um dos valores de ligação, os valores esperados das respectivas classes em F2 e os dados obtidos estão reunidos no quadro N.2. Neste verifica-se que o valôr de ligação que melhor satisfaz é aquele dado pela fórmula de produto com um valor de $\chi^{2}$ insignificante (Probabilidade maior que $10 \%$ ). O valor de $\chi^{2}$ encontrado para o caso da fórmula de soma é significante (Probabilidade menor que $1 \%$ ). Conclue-se, portanto, que no presente caso, a melhor determinação do valôr de ligação foi aquela dada pela fórmula de produto.

O quadro N. 3 contém os dados relativos aos desvios dos valôres de ligação de cada espiga do valôr de ligação obtido do total, desvios êsses que, em relação ao êrro $(d / \sigma)$, mostram resultados todos insignificantes (Probabilidades maiores que 5\%.) Portanto, êsse valor deve ser aceito como indicando a posição de y3 no cromossomo 2, em relação ao gen lg1.

$$
2.2 \quad y 3-g l 2
$$

Os resultados em F2 do cruzamento entre a linhagem contendo $y 3$ e o teste para $g l 2$, cromossomo 2 , estão contidos no 
quadro N. 4. A análise de ligação pelas fórmulas de soma e de produto, para cada uma das espigas estudadas, bem como para o total delas, está reunida no quadro N. 5. Feita a análise pelo teste $\chi^{2}$ e calculados os valôres esperados das respectivas classes em F2, para cada um dos valôres de ligação (Quadro N. 6), verificámos que também aqui, como no cruzamento anterior, o valôr de ligação que melhor satisfaz é o dado pela fórmula de produto, com $\chi^{2}$ insignificante (Probabilidade maior que $10 \%)$. A probabilidade do valôr de $\chi 2$ encontrado para o caso da fórmula de soma é significante (menor que $1 \%$ ). A melhor determinação do valôr de ligação é, portanto, aquela dada pela fórmula de produto.

$\mathrm{O}$ quadro N. 7 contém os dados relativos aos desvios dos valôres de ligação de cada uma das espigas do valor obtido do total. Esses desvios em relação ao êrro (d/ $\sigma$ ), mostram valôres todos insignificantes, com probabilidades maiores que $5 \%$. $\mathrm{O}$ valôr de ligação dado pela fórmula de produto deve, porisso, ser aceito como indicando a posição de $y 3$ no cromossomo 2 , em relação ao gen gl2.

$$
2.3 \quad y 3-\lg 1-g l 2
$$

O mesmo cruzamento onde analisámos a relação $y 3$ - gl2 envolveu também o gen $l g 1$, conforme os dados incluidos no quadro N. 4. O valôr de ligação entre $y 3-l g 1$, neste cruzamento, obtido em F2, pela fórmula de produto, está contido no quadro N. 8, juntamente com o valôr para y3 - gi2. O referido quadro inclui também o valôr de ligação entre $l g 1-g l 2$, obtido pela mesma fórmula, o qual confere com o encontrado por outros pesquizadores (3).

O valôr de ligação obtido neste cruzamento, entre y3 $l g 1$, difere daquele encontrado no cruzamento analisado em 2.1, porém essa diferença não é estatísticamente significante. Analisando o conjunto das 17 espigas, sendo 7 obtidas no cruzamento estudado em 2.1 e 10 no cruzamento aqui analisado, encontrámos, pela fórmula de produto, para êsses dois gens, o valôr de ligação que está contido no quadro N. 9 .

$\mathrm{Da}$ análise dos dados referidos conclui-se que o valôr relativo entre $y 3-\lg 1$ deve ser 7 e que a sequência, no cromossomo 2, deve ser y3 7 lg1 18 gl2.

\section{3 - DISCUSSAO}

$O$ gen $Y 3$, complementar de $Y 1$ e $Y 7$ para coloração amarela do endosperma da semente de milho, foi localizado no grupo 2 por PERRY e SPRAGUE (13) e a sua posição relativa 
no cromossomo 2 foi estudada neste trabalho. $O$ alelo recessivo y3 condiciona, além de ausência de carotinoides no endosperma, plantinhas albescentes, caráter êste atribuido primeiraménte ao gen al ( 9 e 14).

Os resultados analisados na presente publicação, obtidos em F2, permitem constatar que a distância relativa entre os gens y3 e lg1, no cromossomo 2, é de 7 unidades. A posição do gen $\lg 1$, entre $y 3$ e $g l 2$, infere-se dos dados obtidos.

Não foi possível analisar a posição relativa dos três gens em questão na retrocruza, porquanto, só foram obtidas, num total de 3209 plantas, apenas 3 com todos os caracteres recessivos (y3 lg1 gl2). Destas 3, uma morreu logo de início e as duas outras, apesar de terem sido transportadas para estufa e controladas com grande cuidado, não lograram se.desenvolver.

O gen y3 está apenas a 7 unidades do gen $l g 1$ e o gen gl2 apenas a 18 unidades de lg1. Para distâncias relativas tão curtas, a dupla troca de partes é pràticamente nula, devendo ser, portanto, mínimo o valôr da coincidência e máximo o da interferência. $O$ cruzamento realizado com os três gens citados envolveu plantas com as constituições $y 3++\mathrm{x}+\lg 1$ gl2. Assim, para o gen y3 estar entre os gens $\lg 1$ e gl2, seria necessário dupla troca de partes, o que parece pouco provável, com as distâncias relativas mencionadas, para o aparecimento do triplo recesso y3 $\lg 1$ gl2. Estando, porém, o gen $\lg 1$ entre y3 e gl2, conforme indicado nos cruzamentos estudados, a combinação que deveria aparecer por dupla troca seria y3 $\lg 1+$. De fato. esta foi a única combinação que não foi constatada uma só vez siquer. (Quadro N. 4).

\section{4 - CONCLUSÕES}

Os dados analisados no presente trabalho indicam que o fator genético $Y 3$, complementar de $Y 1$ e $Y 7$ para a coloração amarela do endosperma da semente de milho, encontra-se a 7 unidades do gen $l g 1$, no cromossomo 2 . Os cruzamentos realizados, envolvendo os gens $y 3, l g 1$ e $g l 2$ mostraram que a sequência relativa dêsses gens no cromossomo citado é a seguinte : y3 $7 \lg 118 \mathrm{gl2}$.

\section{5 - ABSTRACT}

This paper deals with the genetic relations among y3$\lg 1$ - gl2. The data obtained indicate the relative order in chromosome 2 as follows : y3 $7 \mathrm{gl1} 18 \mathrm{gl2}$. 


\section{6 - BIBLIOGRAFIA}

1) ANDRES, J. Ma. (1939) Analisis Genetico del Color de Endosperma em Algunos Maices Comerciales Argentino. Facultad de Agronomia y Veterinaria de Buenos Aires. Tomo I : 1-25.

2) EMERSON, R. A. (1929) The Genetic Relations of Plant Color in Maize. Cornell University Agricultural Experiment Station Memoir 39 : 1-156.

3) EMERSON, R. A., G. W. BEADLE e A. C. FRASER (1935) A Summary of Linkage Studies in Maize. Cornell University Agricultural Experiment Station Memoir 180: 1-83.

4) GRANER, E. A. (1943) Genética da Côr Amarelo-laranja nas Sementes de Milho. Revista de Agricultura 18 : 443-445.

5) GRANER, E. A. (1945) The Yellow-orange Endosperm of Maize. The American Naturalist 79 : 187-192.

6) GRANER, E. A. (1946) Testes para a Localização de Fatôres Genéticos no Milho. Revista de Agricultura 21: 8-20.

7) GRANER, E. A. (1947) Gen Y7, Complementar de $Y 1$ e Y3 para a Coloroção Amarelo-laranja da Semente de Milho. Revista de Agricultura 22 : 42-54.

8) GRANER, E. A. e W. R. ACCORSI (1949) Os Gens y3-al (plantas albescentes) e y7 (plantas albinas) do Milho e suas Relações com os Plastidios. Scientia Genética 3: 160-171.

9) GRANER, E. A. (1953) Elementos de Genética. Comp. Melhoramentos de S. Paulo.

10) GRANER, E. A. (1950) Genética da Colocação Amarela das Sementes de Milho. Tese. Escola Superior de Agricultura "Luiz de Queiroz".

11) GRANER, E. A. (1952) Como Aprender Estatística. Comp. Melhoramentos de S. Paulo.

12) IMMER, F. R. (1930) Formulae and Tables for Calculating Linkage Intensities. Genetics $15: 81-98$.

13) KOBAL, N. (1951) Estudos sôbre a Genética de Coloração no Endosperma das Sementes de Milho. Tese de Doutoramento. Escola Superior de Agricultura "Luiz de Queiroz".

14) PERRY, H. S. and G. F. SPRAGUE (1936) A Second Chromosome Gene Y3, Producing Yellow Endosperm Color in Maize. Journal of American Society of Agronomy 28 : 990-995.

15) PHIPPS, I. F. (1929) Inheritance and Linkage Relations of Virescent Seedlings in Maize. Cornell University Agricultural Experiment Station Memoir 125: 1-63. 


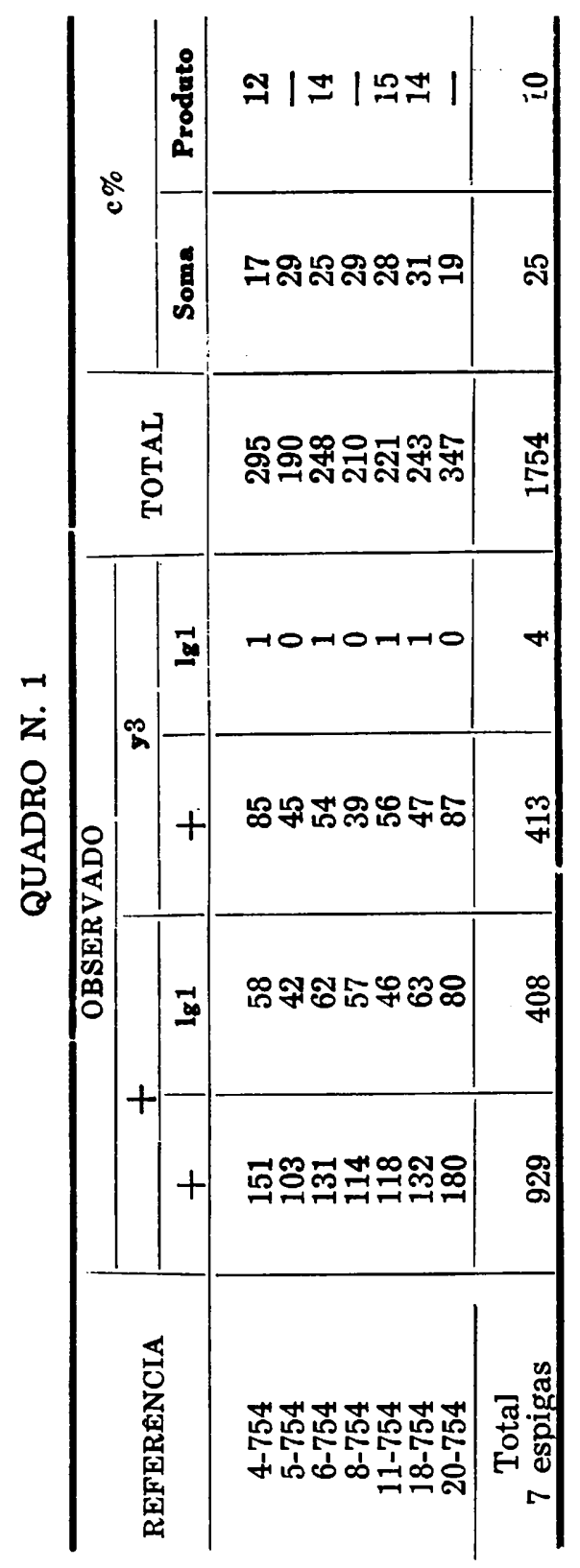


QLADRO N. N. 2

\begin{tabular}{|c|c|c|c|c|c|c|c|c|c|c|c|c|c|c|c|c|c|}
\hline \multirow{2}{*}{ 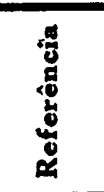 } & \multicolumn{4}{|c|}{ OBSERVADO } & \multirow{2}{*}{ TOTAL } & \multicolumn{2}{|c|}{$\mathrm{c} \%$} & \multicolumn{4}{|c|}{ ESPERADO } & \multicolumn{5}{|c|}{$\chi^{2}$} & \multirow{2}{*}{$\mathbf{P}$} \\
\hline & ++ & $y^{3}+$ & $+\lg 1$ & y3 $\lg 1$ & & Soma & Produto & $t+$ & $\mathbf{y} 3+$ & $+\lg 1$ & y3 $\lg 1$ & \multicolumn{4}{|c|}{ PARCIAIS } & Total & \\
\hline 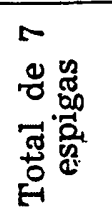 & 929 & 408 & 413 & 4 & 1754 & - & 10 & $\begin{array}{r}904,36 \\
881,38\end{array}$ & $\begin{array}{l}411,14 \\
434,11\end{array}$ & $\begin{array}{l}411,14 \\
434,11\end{array}$ & 27,36 & $\begin{array}{l}0,725 \\
2,570\end{array}$ & $\begin{array}{l}0,008 \\
1,569\end{array}$ & $\begin{array}{l}0,024 \\
1,025\end{array}$ & $\begin{array}{r}20,013 \\
0,033\end{array}$ & $\begin{array}{r}20,770 \\
5,197\end{array}$ & $\begin{array}{l}<0,001 \\
>00,10\end{array}$ \\
\hline
\end{tabular}

QUADRO N. 3

\begin{tabular}{c|c|c|c|c|c}
\hline \multirow{2}{*}{ REFERENCIA } & \multicolumn{2}{|c|}{$\mathrm{c} \%$} & \multirow{2}{*}{ Desvio } & $\sigma$ & $\mathrm{d} / \sigma$ \\
\cline { 2 - 6 } & Individual & Total & & & \\
\hline $4-754$ & 12 & 10 & 2 & 5,71 & 0,35 \\
$6-754$ & 14 & 10 & 4 & 6,21 & 0,64 \\
$11-754$ & 15 & 10 & 5 & 6,53 & 0,76 \\
$18-754$ & 14 & 10 & 4 & 6,25 & 0,64 \\
\hline
\end{tabular}

QUADRO N. 4

\begin{tabular}{|c|c|c|c|c|c|c|c|c|c|}
\hline \multirow{3}{*}{ REF'ERENCIA } & \multicolumn{4}{|c|}{+} & \multicolumn{4}{|c|}{ y3 } & \multirow{3}{*}{ TOTAL } \\
\hline & \multicolumn{2}{|c|}{$t$} & \multicolumn{2}{|c|}{$\mathrm{g} 12$} & \multicolumn{2}{|c|}{ \pm} & \multicolumn{2}{|c|}{$\mathbf{g 1 2}$} & \\
\hline & $t$ & $1 \mathrm{~g} 1$ & \pm & $\lg 1$ & + & $\lg 1$ & + & $\lg 1$ & \\
\hline $\begin{array}{r}3-151 \\
5-151 \\
8-151 \\
13-151 \\
1-152 \\
2-152 \\
6-152 \\
7-152 \\
99-152 \\
13-152 \\
\end{array}$ & $\begin{array}{l}112 \\
105 \\
134 \\
161 \\
151 \\
118 \\
116 \\
120 \\
131 \\
193 \\
\end{array}$ & $\begin{array}{l}20 \\
20 \\
26 \\
32 \\
33 \\
20 \\
23 \\
28 \\
28 \\
34 \\
\end{array}$ & $\begin{array}{l}30 \\
15 \\
13 \\
29 \\
22 \\
19 \\
22 \\
16 \\
27 \\
18 \\
\end{array}$ & $\begin{array}{l}51 \\
53 \\
49 \\
61 \\
56 \\
49 \\
54 \\
35 \\
55 \\
67\end{array}$ & $\begin{array}{r}78 \\
82 \\
91 \\
95 \\
91 \\
69 \\
61 \\
63 \\
73 \\
117 \\
\end{array}$ & $\begin{array}{l}0 \\
0 \\
0 \\
0 \\
0 \\
0 \\
0 \\
0 \\
0 \\
0\end{array}$ & $\begin{array}{l}2 \\
5 \\
0 \\
4 \\
6 \\
4 \\
6 \\
5 \\
4 \\
4 \\
\end{array}$ & $\begin{array}{l}0 \\
0 \\
0 \\
0 \\
1 \\
0 \\
0 \\
1 \\
1 \\
0 \\
\end{array}$ & $\begin{array}{l}293 \\
280 \\
313 \\
382 \\
360 \\
279 \\
282 \\
268 \\
319 \\
433 \\
\end{array}$ \\
\hline $\begin{array}{l}\text { Total } \\
10 \text { espigas }\end{array}$ & 1341 & 264 & 211 & 530 & 820 & 0 & 40 & 3 & 3209 \\
\hline
\end{tabular}


QUADRO N. 5

\begin{tabular}{c|c|c}
\hline \multirow{2}{*}{ REFERENCIA } & \multicolumn{2}{|c}{ c\% } \\
& Soma & g12 \\
\hline $3-151$ & - & Produto \\
$5-151$ & 14 & 14 \\
$8-151$ & 3 & 22 \\
$13-151$ & 24 & 20 \\
$1-152$ & 14 & 27 \\
$2-152$ & 17 & 23 \\
$6-152$ & 39 & 27 \\
$7-152$ & 17 & 24 \\
$9-152$ & 26 & 20 \\
$13-152$ & & \\
\hline TOTAL & 17 & 22 \\
\hline
\end{tabular}

QUADRO N. 6

\begin{tabular}{|c|c|c|c|c|c|c|c|c|c|c|c|c|c|c|c|c|c|}
\hline \multirow{2}{*}{ 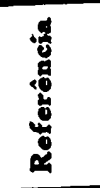 } & \multicolumn{4}{|c|}{ OBSERVADO } & \multirow{2}{*}{ TOTAL } & \multicolumn{2}{|c|}{$\%$} & \multicolumn{4}{|c|}{ ESPERADO } & \multicolumn{5}{|c|}{$\chi^{2}$} & \multirow{2}{*}{$\mathbf{P}$} \\
\hline & ++ & $83+$ & +812 & $y^{3}-g 12$ & & Soma & Produto & ++ & $y^{3}+$ & $+\mathrm{s} 12$ & y3 812 & & PAF & & & Total & \\
\hline 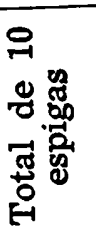 & 1605 & 820 & 741 & 43 & 3209 & - & - & $\begin{array}{r}1627,60 \\
1643,33\end{array}$ & $\begin{array}{l}779,14 \\
763,42\end{array}$ & $\begin{array}{l}779,14 \\
763,42\end{array}$ & 38,83 & 0,89 & 4,19 & 0,66 & $\begin{array}{r}16,98 \\
0,45\end{array}$ & 21,30 & $\begin{array}{l}<0,001 \\
>0,10\end{array}$ \\
\hline
\end{tabular}




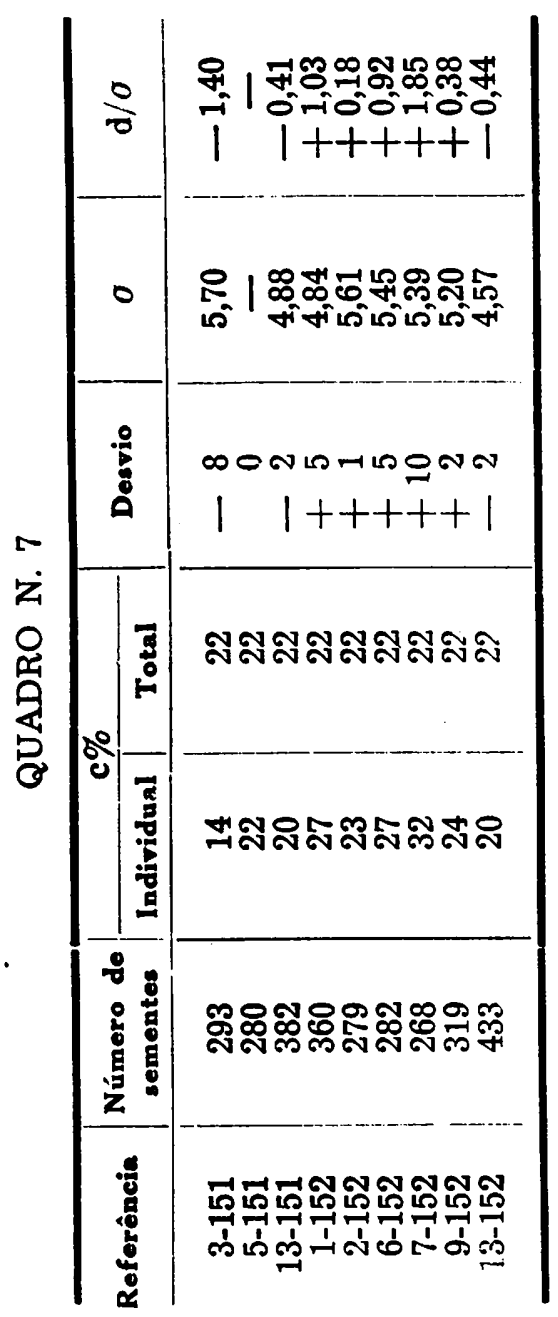


QUADRO N. 8

\begin{tabular}{c|c|c|c}
\hline $\begin{array}{c}\text { Total de } 10 \\
\text { eapigas }\end{array}$ & $\mathrm{y} 3-\lg 1$ & $\lg 1-g 12$ & $\mathrm{J3}-\mathrm{gl2}$ \\
\hline $\begin{array}{c}\text { c\% } \\
\text { (Produto) }\end{array}$ & 6 & 18 & 22 \\
\hline
\end{tabular}

QUADRO N. 9

\begin{tabular}{c|c|c|c|c|c}
\hline $\begin{array}{c}\text { Total de } \\
17 \text { eapigas }\end{array}$ & +2 & \multicolumn{2}{|c|}{$+\mathrm{s}^{3}$} & $c \%$ \\
\hline 4963 & 2481 & 1202 & 1273 & 7 & 7 \\
\hline
\end{tabular}

